\title{
Mitochondrial energetics in liver and skeletal muscle after energy restriction in young rats
}

\author{
Raffaella Crescenzo ${ }^{1}$, Francesca Bianco ${ }^{1}$, Italia Falcone ${ }^{1}$, Paola Coppola ${ }^{1}$, Abdul G. Dulloo ${ }^{2}$, \\ Giovanna Liverini ${ }^{1}$ and Susanna Iossa ${ }^{1 *}$ \\ ${ }^{1}$ Department of Structural and Functional Biology, University of Naples, Complesso Universitario di Monte Sant'Angelo, \\ Edificio 7, Via Cinthia-I-80126, Napoli, Italy \\ ${ }^{2}$ Department of Medicine, Division of Physiology, University of Fribourg, Switzerland
}

(Submitted 18 March 2011 - Final revision received 26 September 2011 - Accepted 30 September 2011 - First published online 16 November 2011)

\section{Abstract}

The present study investigated the effect of 2 weeks of energy restriction on whole body, liver and skeletal muscle energy handling. We measured whole-body oxygen consumption, as well as mitochondrial protein mass, respiratory capacity and energetic coupling in liver and skeletal muscle from food-restricted (FR) rats, age- and weight-matched controls. We also assessed markers of oxidative damage and antioxidant defences. The present results show that, in response to energy restriction, an adaptive decrease in whole-body energy expenditure is coupled with structural and functional changes in mitochondrial compartment, both in liver and skeletal muscle. In fact, liver mitochondrial mass per g of liver significantly increased, whereas total hepatic mitochondrial oxidative capacity was lower in FR than in control rats, because of a significant decrease in liver contribution to total body weight. In skeletal muscle, sub-sarcolemmal (SS) mitochondrial respiratory capacity, as well as SS and inter-myofibrillar (IMF) mitochondrial protein mass per g of tissue, was significantly lower in FR rats, compared to controls. Finally, a decrease in oxidative damage was found in liver but not in skeletal muscle mitochondria from FR rats, whereas an increase in antioxidant defence was found in both tissues. From the present results, it appears that skeletal muscle is involved in the decrease in energy expenditure induced by energy restriction. Energy sparing is achieved through changes in the activity (SS), mass (SS and IMF) and efficiency (IMF) of mitochondrial compartment.

\section{Key words: Energy restriction: Mitochondrial efficiency: Respiratory quotient: RMR}

It is well demonstrated that in response to a period of limited supply of energy and nutrients (energy restriction), an adaptive decrease in whole-body energy expenditure occurs, and this is partly attributable to an increase in metabolic efficiency $^{(1)}$. This adaptation allows the organism to spare energy, thus slowing the rate of depletion of lipid stores ${ }^{(2)}$. This energy conservation mechanism, which probably evolved as an adaptive survival response to periodic food shortage, is thought to be an important factor that contributes to the failure of most dietary regimens in slimming therapies. Energy restriction is also known to increase lifespan in a wide range of species and to delay in humans the onset of agerelated chronic diseases such as heart disease and stroke ${ }^{(3)}$, probably through reduced oxidative damage to macromolecules $^{(4)}$. Both the aforementioned processes seem to involve alterations in mitochondrial functions ${ }^{(5,6)}$. In fact, the main cellular compartment involved in the control of energy transduction efficiency is represented by mitochondria, which are able to convert energy from fuels into ATP through oxidative phosphorylation, whose efficiency depends on the degree of coupling between oxygen consumption $\left(\mathrm{VO}_{2}\right)$ and ATP synthesis. The degree of coupling can vary according to the metabolic needs of the cell ${ }^{(7)}$, and is regulated by the amount of cellular unbound fatty acids that can act as natural uncouplers of oxidative phosphorylation ${ }^{(8,9)}$. In addition, the mitochondria are the main producers of reactive oxygen species (ROS), and are also highly exposed to oxidative stress ${ }^{(10)}$.

As many studies have investigated preferentially long-term (months) effect of energy restriction in adult rats, although information on the early adaptive phase to decreased energy intake is scarce, especially in growing rats, we investigated the effect of 2 weeks of energy restriction in 7-week-old rats by evaluating mitochondrial efficiency and oxidative status

\footnotetext{
Abbreviations: $\eta$, optimal thermodynamic efficiency; AM, age-matched control rats; BSA, bovine serum albumin; FR, food restricted; $\boldsymbol{g}_{\text {av }}, \boldsymbol{g}_{\text {average; }}$ IMF, intermyofibrillar; $q$, thermodynamic coupling; ROS, reactive oxygen species; RQ, respiratory quotient; SOD, superoxide dismutase; SS, sub-sarcolemmal; VCO 2 , carbon dioxide production; $\mathrm{VO}_{2}$, oxygen consumption; WM, weight-matched control rats.
} 
in the skeletal muscle and liver, which together account for about $50 \%$ of whole-body metabolic rate ${ }^{(11)}$. With respect to skeletal muscle, analyses were carried out taking into account that the mitochondrial population is heterogeneous, with mitochondria located either beneath the sarcolemmal membrane (sub-sarcolemmal, SS) or between the myofibrils (inter-myofibrillar, IMF) $^{(12)}$. It is important that both the SS and IMF mitochondrial populations are studied because they exhibit different energetic characteristics and therefore can be differently affected by physiological stimuli ${ }^{(13-15)}$. Furthermore, in order to integrate the findings about mitochondrial energetics with changes in whole-body energy handling induced by energy restriction, we concomitantly studied whole-body metabolic characteristics.

\section{Experimental methods}

Animals

Male Sprague-Dawley rats were adapted to room and cage environments for at least $5 \mathrm{~d}$ before the start of the experiment. The rats were caged singly in a temperature-controlled room $\left(23 \pm 1^{\circ} \mathrm{C}\right)$ with a $12 \mathrm{~h}$ light $-12 \mathrm{~h}$ dark cycle and were maintained on a commercial pelleted chow diet (Mucedola, Settimo Milanese, Italy) consisting, by energy, of $29 \%$ protein, $60 \%$ carbohydrates and $11 \%$ fat, and had free access to tap water. The experiments were conducted after this period of adaptation in rats selected on the basis of body weight being within $\pm 5 \mathrm{~g}$ of the mean body weight. Animals used in the present studies were maintained in accordance with the Italian Health Ministry regulations and guidelines for the care and use of laboratory animals. All experimental procedures involving animals were approved by 'Comitato Etico-Scientifico per la Sperimentazione Animale' of the University 'Federico II' of Naples.

\section{Experimental design}

A group composed of 7-week-old rats, with a body weight of 230 (SEM 5) g, was food restricted (FR) for $14 \mathrm{~d}$ at approximately $50 \%$ of their ad libitum food intake, corresponding to $165 \mathrm{~kJ}$ metabolisable energy/d. Restriction was carried out using the earlier pelleted chow diet, but ensuring appropriate micronutrient supply. Such a reduction in food intake leads to growth arrest, as shown by the fact that at the end of this period, body weight of the rats was 233 (sem 2) g. A second group composed of 7-week-old rats was fed ad libitum with the same diet during all the period of treatment and represented the age-matched (AM) control. A third group composed of 5-week-old rats was fed ad libitum during all the period of treatment and represented the weight-matched (WM) control, as these rats exhibited a body weight similar to that of FR rats at the end of the experimental period. During the whole experimental period, metabolisable energy intake was monitored as detailed previously ${ }^{(16)}$.

The day before the killing of the animals, $24 \mathrm{~h} \mathrm{VO}_{2}$ and carbon dioxide production $\left(\mathrm{VCO}_{2}\right)$ were recorded and urine was collected for the whole $(24 \mathrm{~h})$ period. The animals were killed in the post-absorptive phase by decapitation and used for blood collection and liver and hind leg skeletal muscle harvesting or for body composition measurements.

The $24 \mathrm{~h}$ oxygen consumption, carbon dioxide production, general physical activity, respiratory quotient, urinary nitrogen and non-protein respiratory quotient

The $24 \mathrm{~h} \mathrm{VO}_{2}, \mathrm{VCO}_{2}$, general physical activity and respiratory quotient (RQ) of the rats were recorded with a monitoring system (Panlab s.r.l., Cornella, Barcelona, Spain) composed of four-chamber indirect open-circuit calorimeter, designed for the continuous monitoring of up to four rats simultaneously. Measurements were performed every $15 \mathrm{~min}$ for $3 \mathrm{~min}$ in each cage. Urine was collected for the whole $(24 \mathrm{~h})$ period and urinary nitrogen levels were measured by an enzymatic colorimetric method (FAR s.r.l., Settimo di Pescantina, Verona, Italy). The $24 \mathrm{~h}$ non-protein RQ was then calculated.

\section{Blood parameters}

Blood was collected in EDTA tubes and plasma was obtained by centrifugation at $1400 \boldsymbol{g}_{\text {average }}\left(\boldsymbol{g}_{\text {av }}\right)$ for $8 \mathrm{~min}$ at $4{ }^{\circ} \mathrm{C}$. Serum NEFA concentrations were measured by colorimetric enzymatic method using commercial kits (Roche Diagnostics, Mannheim, Germany).

\section{Determination of whole body, skeletal muscle and liver composition}

The guts were cleaned of undigested food and the whole carcasses were autoclaved. After dilution (1:2 distilled water) and subsequent homogenisation with a Polytron homogeniser (Kinematica, Luzern, Switzerland), the resulting homogenates were aliquoted and frozen at $-20^{\circ} \mathrm{C}$ until the day of measurements.

Duplicate samples of the homogenised carcass were analysed for energy content by bomb calorimetry and for fat content by the Folch extraction method ${ }^{(17)}$. Body protein was determined from a general formula relating energy derived from fat, total energy value of the carcass and energy derived from protein ${ }^{(18)}$; the energy values for body fat and protein were taken as 38.6 and $22.7 \mathrm{~kJ} / \mathrm{g}$, respectively ${ }^{(19)}$. Whole-body and liver water content was determined by drying samples in an oven maintained at $70^{\circ} \mathrm{C}$ for $48 \mathrm{~h}$.

Liver glycogen content was assessed by direct enzymatic procedure $^{(20)}$. Finally, lipid content in liver and skeletal muscle was gravimetrically assessed after extraction by the Folch method ${ }^{(17)}$.

\section{Preparation of liver- and skeletal muscle-isolated mitochondria, and measurements of mitochondrial respiration, thermodynamic coupling and efficiency}

Liver and hind leg muscles were rapidly removed and used for the preparation of homogenates and isolated mitochondria, as described previously ${ }^{(21,22)}$. Briefly, liver tissue fragments were gently homogenised with a medium containing 
220 mm-mannitol, 70 mm-sucrose, 20 mm-HEPES, 1 mm-EDTA and $0.1 \%(\mathrm{w} / \mathrm{v})$ fatty acid-free bovine serum albumin (BSA; $\mathrm{pH}$ 7.4) in a Potter Elvehjem homogeniser set at $500 \mathrm{rpm}$ (four strokes per min). The homogenate was then centrifuged at $1000 \boldsymbol{g}_{\text {av }}$ for $10 \mathrm{~min}$, and the resulting supernatant was again centrifuged at $3000 \boldsymbol{g}_{\mathrm{av}}$ for $10 \mathrm{~min}$. The mitochondrial pellet was washed twice and finally re-suspended in a medium containing $80 \mathrm{~mm}-\mathrm{LiCl}, 50 \mathrm{~mm}$-HEPES, $5 \mathrm{~mm}$-Tris-PO $4,1 \mathrm{~mm}-$ ethylene glycol tetraacetic acid (EGTA) and $0 \cdot 1 \%(\mathrm{w} / \mathrm{v})$ fatty acid-free BSA ( $\mathrm{pH} 7 \cdot 0)$. Control experiments of enzymatic and electron microscopy characterisation have shown that isolation procedure of the present study (centrifugation at $3000 \boldsymbol{g}_{\text {av }}$ for $10 \mathrm{~min}$ ) results in a cellular fraction essentially constituted by mitochondria.

Hind leg muscles were freed of excess fat and connective tissue, finely minced, washed in a medium containing

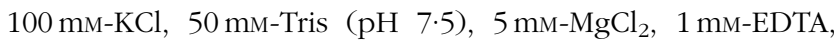
$5 \mathrm{~mm}$-EGTA and $0 \cdot 1 \%(\mathrm{w} / \mathrm{v})$ fatty acid-free BSA and homogenised with the aforementioned medium (1:8, w/v) at $500 \mathrm{rpm}$ (four strokes per min). Homogenate was then centrifuged at $500 \boldsymbol{g}_{\text {av }}$ for $10 \mathrm{~min}$ and the resulting precipitate was subsequently used for the preparation of the IMF mitochondria. The supernatant was centrifuged at $3000 \boldsymbol{g}_{\text {av }}$ for $10 \mathrm{~min}$ and the pellet containing SS mitochondria was washed twice and re-suspended in suspension medium (250 mm-sucrose, $50 \mathrm{~mm}$-Tris ( $\mathrm{pH} 7.5$ ) and $0.1 \%$ fatty acid-free BSA). The pellet from the $500 \boldsymbol{g}$ centrifugation was re-suspended in a small amount of homogenisation solution and treated with protease nagarse $(3.6 \mathrm{U} / \mathrm{g}$ tissue) for $4 \mathrm{~min}$. The suspension was then homogenised and centrifuged at $3000 \boldsymbol{g}_{\mathrm{av}}$ for $10 \mathrm{~min}$. The resulting supernatant was rapidly discarded and the pellet was re-suspended and centrifuged at $500 \boldsymbol{g}_{\text {av }}$ for $10 \mathrm{~min}$. The supernatant containing the IMF mitochondria was centrifuged at $3000 \boldsymbol{g}_{\text {av }}$ for $10 \mathrm{~min}$ and the pellet was washed once and re-suspended in suspension medium. Contamination of SS and IMF mitochondria by other ATPasecontaining membranes was $<10 \%$, and nagarse treatment of SS mitochondria had no effect on respiratory activities of states 3 and 4 .

$\mathrm{VO}_{2}$ rate was measured polarographically with a Clark-type electrode (Yellow Springs Instruments; Yellow Springs, $\mathrm{OH}$, USA) in a $3 \mathrm{ml}$ glass cell, at a temperature of $30^{\circ} \mathrm{C}$. Hepatic homogenates or isolated mitochondria were incubated in a medium containing $80 \mathrm{~mm}-\mathrm{KCl}, 50 \mathrm{~mm}$-HEPES, $5 \mathrm{~mm}-\mathrm{K}_{2} \mathrm{HPO}_{4}$, $1 \mathrm{~mm}-\mathrm{EGTA}$ and $0 \cdot 1 \%(\mathrm{w} / \mathrm{v})$ fatty acid-free BSA ( $\mathrm{pH} 7 \cdot 0)$, whereas skeletal muscle homogenates or isolated SS or IMF mitochondria were incubated in a medium containing

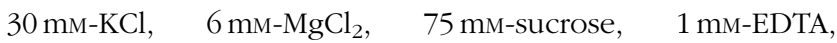
$20 \mathrm{~mm}-\mathrm{KH}_{2} \mathrm{PO}_{4}(\mathrm{pH} 7 \cdot 0)$ and $0 \cdot 1 \%(\mathrm{w} / \mathrm{v})$ fatty acid-free BSA $(\mathrm{pH} 7 \cdot 0)$. All samples were allowed to oxidise their endogenous substrates for $3 \mathrm{~min}$. Either $10 \mathrm{~mm}$-glutamate $+2.5 \mathrm{~mm}$-malate; or $40 \mu \mathrm{m}$-palmitoyl-coenzyme $\mathrm{A}+2 \mathrm{~mm}$-carnitine $+2.5 \mathrm{~mm}-$ malate; or succinate $10 \mathrm{~mm}+$ rotenone $3.75 \mu \mathrm{m}$ were added as substrate. State $3 \mathrm{VO}_{2}$ was measured in the presence of $0.3 \mathrm{~mm}$-ADP. State 4 respiration was obtained in the presence of oligomycin $(4 \mu \mathrm{g} / \mathrm{ml})$ in the homogenates or in the absence of ADP in isolated mitochondria. Respiratory control ratio was always calculated as the ratio between states 3 and 4 .
The degree of thermodynamic coupling $(q)$ was determined in liver, SS and IMF mitochondria by applying equation 11 by Cairns et al. ${ }^{(23)}: q=\sqrt{1-\left(J_{\mathrm{o}}\right)_{\mathrm{sh}} /\left(J_{\mathrm{o}}\right)_{\text {unc }}}$, where $\left(J_{\mathrm{o}}\right)_{\mathrm{sh}}$ represents the $\mathrm{VO}_{2}$ rate in the presence of oligomycin that inhibits ATP synthase and $\left(J_{\mathrm{o}}\right)_{\mathrm{unc}}$ is the uncoupled rate of $\mathrm{VO}_{2}$ induced by carbonyl cyanide 4-(trifluoromethoxy) phenylhydrazone (FCCP), which dissipates the transmitochondrial proton gradient. $\left(J_{\mathrm{o}}\right)_{\mathrm{sh}}$ and $\left(J_{\mathrm{o}}\right)_{\mathrm{unc}}$ were measured as earlier using succinate $10 \mathrm{~mm}+$ rotenone $3.75 \mu \mathrm{m}$ in the presence of oligomycin $2 \mu \mathrm{g} / \mathrm{ml}$ or FCCP $1 \mu \mathrm{M}$, respectively, both in the absence and in the presence of palmitate at a concentration of $50 \mu \mathrm{m}$ for liver, $45 \mu \mathrm{M}$ for SS and $65 \mu \mathrm{M}$ for IMF mitochondria.

The optimal thermodynamic efficiency $(\eta)$ of oxidative phosphorylation was calculated by using equation 13 by Cairns et al. ${ }^{(23)}$ :

$$
\eta=\frac{q^{2}}{\left(1+\sqrt{\left(1-q^{2}\right)}\right)^{2}} .
$$

\section{Cytochrome oxidase-specific activity and cytochrome c mitochondrial content}

Cytochrome oxidase-specific activity was measured polarographically $^{(24)}$. Aliquots of homogenates or isolated mitochondria were incubated for $30 \mathrm{~min}$ at $0^{\circ} \mathrm{C}$ in a medium containing $1 \mathrm{~mm}$-ATP, $50 \mathrm{~mm}$-HEPES, $100 \mathrm{~mm}-\mathrm{KCl}, \quad 5 \mathrm{~mm}$ $\mathrm{MgCl}_{2}, 1 \mathrm{~mm}$-EDTA, $5 \mathrm{~mm}$-EGTA and $1.5 \mathrm{mg} / \mathrm{ml}$ lubrol $(\mathrm{pH}$ 7.0) to solubilise membranes. At the end of the incubation period, $\mathrm{VO}_{2}$ was measured in a medium containing $75 \mathrm{~mm}$ HEPES, $30 \mu \mathrm{m}$-cytochrome $c, 10 \mathrm{~mm}$-malonate, $4 \mu \mathrm{m}$-rotenone, $0.5 \mathrm{~mm}$-dinitrophenol, $4 \mathrm{~mm}$-ascorbate and $0.3 \mathrm{~mm}-\mathrm{N}, \mathrm{N}, \mathrm{N}^{\prime}, \mathrm{N}^{\prime}-$ tetramethyl-P-phenylenediamine (TMPD).

Mitochondrial mass was evaluated by Western blot analysis of cytochrome $c$, as described previously ${ }^{(25)}$.

\section{Aconitase-specific activity, lipid peroxidation and superoxide dismutase-specific activity}

Aconitase-specific activity was measured spectrophotometrically by the method of Gardner ${ }^{(26)}$, lipid peroxidation was determined according to Fernandes et $a l^{(27)}$, whereas superoxide dismutase (SOD)-specific activity was measured according to Flohè and Otting ${ }^{(28)}$.

\section{Chemicals}

All chemicals utilised were of analytical grade and were purchased from Sigma (St Louis, MO, USA)

\section{Statistical analysis}

Data are provided as mean values and their standard error of mean. Statistical analyses were performed using one-way ANOVA, followed by the Tukey's test after ANOVA established significant differences. All analyses were performed using GraphPad Prism 4 (GraphPad Software, Inc., San Diego, CA, USA). 


\section{Results}

Body composition measurements show that, at the end of energy restriction, FR rats had lower body energy content and lipid mass than controls, while protein and water mass was the same (Table 1). As for organ weight and composition, FR rats exhibited significantly lower values of liver weight compared to controls, both as absolute values and as expressed per $100 \mathrm{~g}$ body weight. Hepatic water content was significantly higher in FR rats compared to controls when expressed per g of liver, whereas it was significantly lower when expressed per whole liver. In addition, hepatic lipid content was significantly lower in FR rats compared to controls, only when expressed per whole tissue, whereas no variation was found in hepatic glycogen content. Skeletal muscle lipid content and plasma NEFA levels were significantly lower in FR rats compared to controls.

The $24 \mathrm{~h}$ monitoring of $\mathrm{VO}_{2}$ and $\mathrm{VCO}_{2}$ are shown in Fig. 1(A) and (B), respectively. A significant decrease in mean $24 \mathrm{~h} \mathrm{VO}_{2}$ and $\mathrm{VCO}_{2}$ (Fig. 1(C)), mean $24 \mathrm{~h} \mathrm{RQ}$ and non-protein RQ (Fig. 1(D)), as well as in RQ calculated separately for light- and dark-phase (Fig. 1(E)), was found in FR rats compared to controls. Mean values of a $2 \mathrm{~h}$ period (13.00-15.00 hours) were taken as indicative of RMR and they were found to be significantly different in the three groups of rats. In fact, RMR values expressed as $\mathrm{ml}$ of $\mathrm{O}_{2}$ per $\min \times \mathrm{kg}^{0.75}$ were: $\mathrm{WM}=17 \cdot 87$ ( $\left.\mathrm{SEM} 0 \cdot 25\right), \mathrm{AM}=14 \cdot 17$ (SEM 0.45$)$ and $F R=10.88$ (SEM 0.32). No variation was found among groups in light- or dark-phase general physical activity (Fig. 1(F)).

Respiratory capacities of liver, SS and IMF skeletal muscle mitochondria were evaluated in homogenates and isolated organelles by using NAD, FAD and lipid substrates (Figs. 2 and 3). In liver (Fig. 2), state 3 respiratory capacities with glutamate or palmitoyl-CoA as substrate were significantly higher in homogenates from FR rats compared to controls (Fig. 2(A)), whereas in isolated mitochondria, significantly lower values were found in FR rats only with succinate as substrate (Fig. 2(B)). When expressed per $100 \mathrm{~g}$ body weight, hepatic respiratory capacities were significantly lower in FR rats compared to controls (Fig. 2(C)). In skeletal muscle (Fig. 3), significantly lower state 3 respiratory capacities were found in FR rats compared to controls when assessed in homogenates (Fig. 3(A)). In isolated organelles, respiratory capacities with glutamate or succinate as substrate of SS mitochondria were significantly lower in FR rats, compared to controls (Fig. 3(B)), whereas no significant variation was found in IMF mitochondria with any substrate (Fig. 3(C)). No significant variation in state 4 and respiratory control ratio values were found in any condition (data not shown).

Mitochondrial energetic efficiency was assessed by determining the degree of $q$ and the $\eta$. To this end, state 4 respiration in the presence of oligomycin and uncoupled respiration in the presence of FCCP were monitored (Table 2). Measurements were carried out both in the absence and in the presence of physiological concentrations of palmitate, to take into account the uncoupling effect of cellular unbound fatty acids. As for the liver, increased state 4 respiration was found in FR rats compared to controls in the absence of palmitate, whereas in the presence of palmitate, state 4 respiration in FR rats was lower than that in controls. Uncoupled respiration was lower in FR rats compared to controls, both in the absence and in the presence of palmitate. Consequently, $q$ and $\eta$ values were significantly lower in FR rats compared to controls in the absence of palmitate, whereas no variation was found in the presence of palmitate. In skeletal muscle SS mitochondria, uncoupled respiration was significantly lower in FR rats compared to controls, both in the absence and in the presence of palmitate, whereas no variation was found in state 4 respiration. Consequently, $q$ and $\eta$ values were significantly lower in SS mitochondria from FR rats, compared to controls, both in the absence and in the presence of palmitate. As for IMF mitochondria, no variation in any of the earlier parameters was found, both in the presence and in the absence of palmitate.

Table 1. Body composition in food restricted (FR) and control rats (Mean values with their standard errors of six rats per group)

\begin{tabular}{|c|c|c|c|c|c|c|}
\hline & \multicolumn{2}{|c|}{ WM } & \multicolumn{2}{|c|}{ AM } & \multicolumn{2}{|c|}{$\mathrm{FR}$} \\
\hline & Mean & SEM & Mean & SEM & Mean & SEM \\
\hline Body weight (g) & $235^{a}$ & 2 & $366^{b}$ & 4 & $233^{a}$ & 2 \\
\hline Body lipids (\%) & $7.4^{\mathrm{a}}$ & 0.6 & $9 \cdot 1^{\mathrm{a}}$ & 0.8 & $4.7^{\mathrm{b}}$ & 0.3 \\
\hline Body proteins (\%) & $20 \cdot 9^{\mathrm{a}}$ & $0 \cdot 8$ & $19 \cdot 4^{\mathrm{a}}$ & 0.7 & $21 \cdot 1^{\mathrm{a}}$ & 0.5 \\
\hline Body water $(\%)$ & $69 \cdot 5^{a}$ & $3 \cdot 2$ & $66 \cdot 4^{a}$ & $3 \cdot 3$ & $69 \cdot 7^{\mathrm{a}}$ & $2 \cdot 8$ \\
\hline Body energy $(\mathrm{kJ} / \mathrm{g})$ & $7 \cdot 6^{\mathrm{a}}$ & 0.2 & $7 \cdot 9^{\mathrm{a}}$ & 0.3 & $6 \cdot 6^{\mathrm{b}}$ & 0.2 \\
\hline Liver weight (g) & $11.5^{\mathrm{a}}$ & 0.5 & $17 \cdot 9^{\mathrm{b}}$ & $1 \cdot 1$ & $8 \cdot 1^{\mathrm{c}}$ & 0.2 \\
\hline Liver weight ( $\mathrm{g} / 100 \mathrm{~g}$ body weight) & $4.9^{\mathrm{a}}$ & 0.1 & $4.9^{\mathrm{a}}$ & 0.3 & $3.5^{\mathrm{b}}$ & 0.1 \\
\hline Hepatic water (mg/g liver) & $723^{\mathrm{a}}$ & 14 & $738^{a}$ & 12 & $815^{\mathrm{b}}$ & 19 \\
\hline Hepatic water (mg/liver) & $8314^{a}$ & 144 & $13210^{\mathrm{b}}$ & 122 & $6601^{c}$ & 198 \\
\hline Hepatic glycogen (mg/g liver) & $42^{\mathrm{a}}$ & 2 & $40^{\mathrm{a}}$ & 2 & $39^{\mathrm{a}}$ & 3 \\
\hline Hepatic lipids (mg/g liver) & $52^{a}$ & 6 & $47^{\mathrm{a}}$ & 6 & $54^{a}$ & 6 \\
\hline Hepatic lipids (mg/liver) & $649^{\mathrm{a}}$ & 59 & $841^{\mathrm{b}}$ & 50 & $453^{c}$ & 41 \\
\hline Skeletal muscle lipids (mg/g) & $41^{\mathrm{a}}$ & 2 & $39^{a}$ & 1 & $33^{\mathrm{b}}$ & 1 \\
\hline NEFA $(\mathrm{mmol} / \mathrm{l})$ & $0.118^{\mathrm{a}}$ & 0.011 & $0 \cdot 109^{\mathrm{a}}$ & 0.010 & $0.068^{\mathrm{b}}$ & 0.012 \\
\hline
\end{tabular}

WM, weight-matched control rats; AM, age-matched control rats.

a,b,c Mean values within a row with unlike superscript letters were significantly different $(P<0.05$; one-way ANOVA followed by Tukey post-test). 

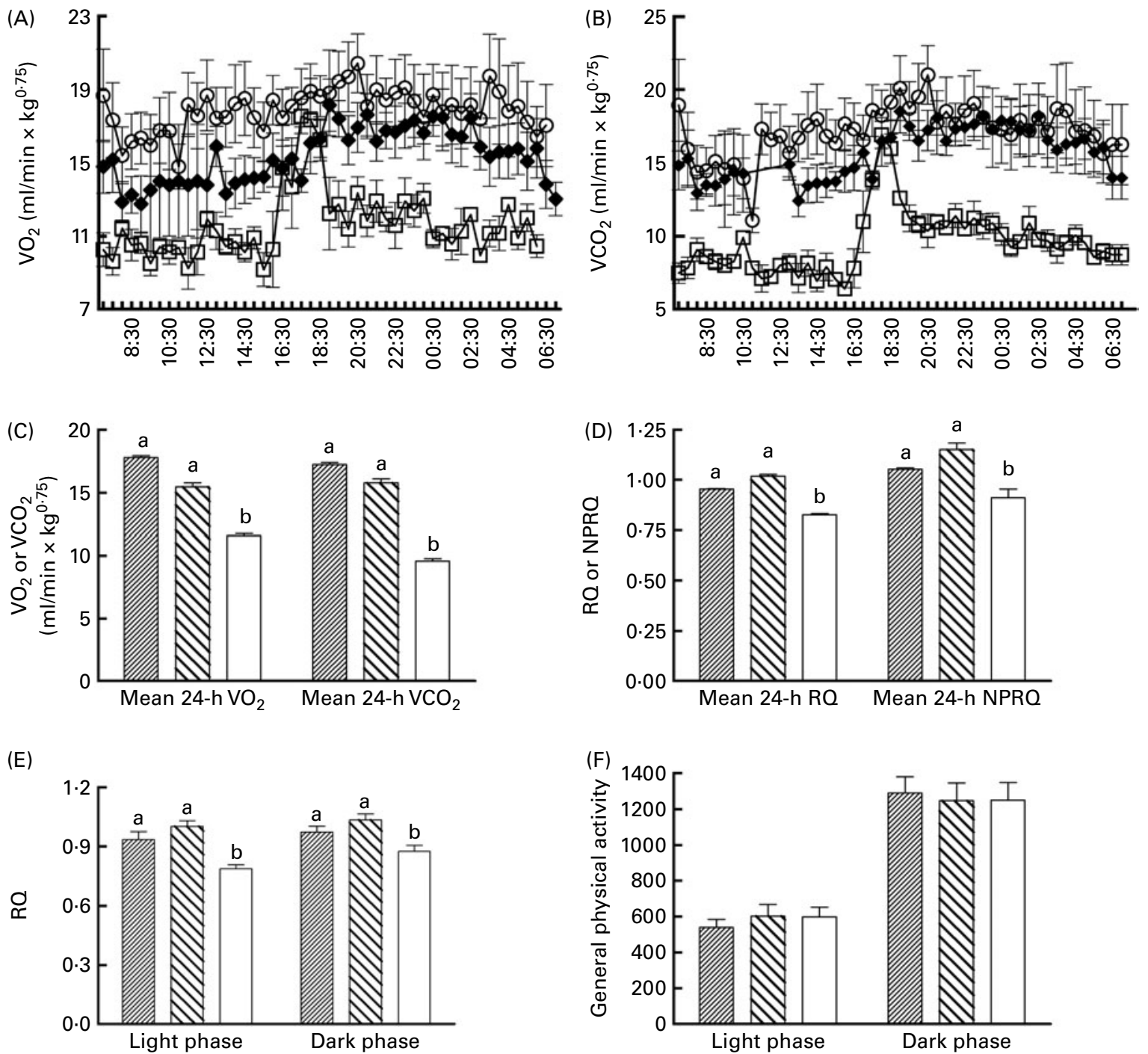

Fig. 1. (A) The $24 \mathrm{~h}$ monitoring of oxygen consumption, $\mathrm{VO}_{2}$, and (B) carbon dioxide production, $\mathrm{VCO}_{2}$, as well as (C) mean $24 \mathrm{~h} \mathrm{VO}_{2}$ and $\mathrm{VCO}_{2}$, (D) mean $24-\mathrm{h}$ respiratory quotient (RQ) and non-protein $R Q(N P R Q),(E)$ light- or dark-phase $R Q$ and $(F)$ light- or dark-phase general physical activity values in food-restricted and control rats. Values are means, with their standard errors represented by vertical bars of six rats per group. ${ }^{\mathrm{a}, \mathrm{b}}$ Bars with unlike letters were significantly different $(P<0.05$; one-way ANOVA followed by Tukey's post-test). WM, weight-matched control rats $((\mathrm{A}, \mathrm{B})-0$; $(\mathrm{C}-\mathrm{F})$ 四); AM, age-matched control rats $((\mathrm{A}$, $\mathrm{B})$ $\rightarrow-;(C-F) \mathbb{\nabla}) ; F R$, food-restricted rats $((A, B)-\square ;(C-F)$ D).

To assess changes in mitochondrial protein mass in FR rats, we utilised three approaches, namely, (1) by measuring homogenate cytochrome $c$ content, (2) by measuring the activity of a mitochondrial marker enzyme cytochrome oxidase in homogenates and isolated mitochondria and (3) by evaluating the mitochondrial yield (i.e. $\mathrm{mg}$ of isolated protein per $\mathrm{g}$ of starting wet tissue) in each mitochondrial sub-population (Table 3). In liver, higher cytochrome $c$ content, as well as higher cytochrome oxidase activity per $g$ of tissue, both in homogenate and isolated mitochondria, were found in FR rats compared to controls. Higher protein yield was found in mitochondria from FR rats compared to controls, whereas no variation was found in cytochrome oxidase recovery and cytochrome oxidase-specific activity per $\mathrm{mg}$ of protein. In skeletal muscle, lower homogenate cytochrome $c$ content, as well as cytochrome oxidase activity per $\mathrm{g}$ of tissue, both in homogenate and in isolated SS and IMF mitochondria, was found in FR rats compared to controls. Lower protein yield was found in IMF and SS mitochondria in FR rats compared to controls, whereas no variation was found in cytochrome oxidase-specific activity per $\mathrm{mg}$ of protein and cytochrome oxidase recovery. Therefore, independently of the approaches utilised, we found that the mitochondrial mass was significantly increased in liver and significantly reduced in both SS and IMF compartments in FR rats, compared to controls.

Aconitase- and SOD-specific activity were measured and taken as an index of cellular oxidative damage and antioxidant defences, respectively (Fig. 4). In liver, a significant increase in aconitase and SOD activity was found in FR rats, compared to controls. In skeletal muscle, a significant increase in SOD activity was found in IMF and SS mitochondria from FR rats, 

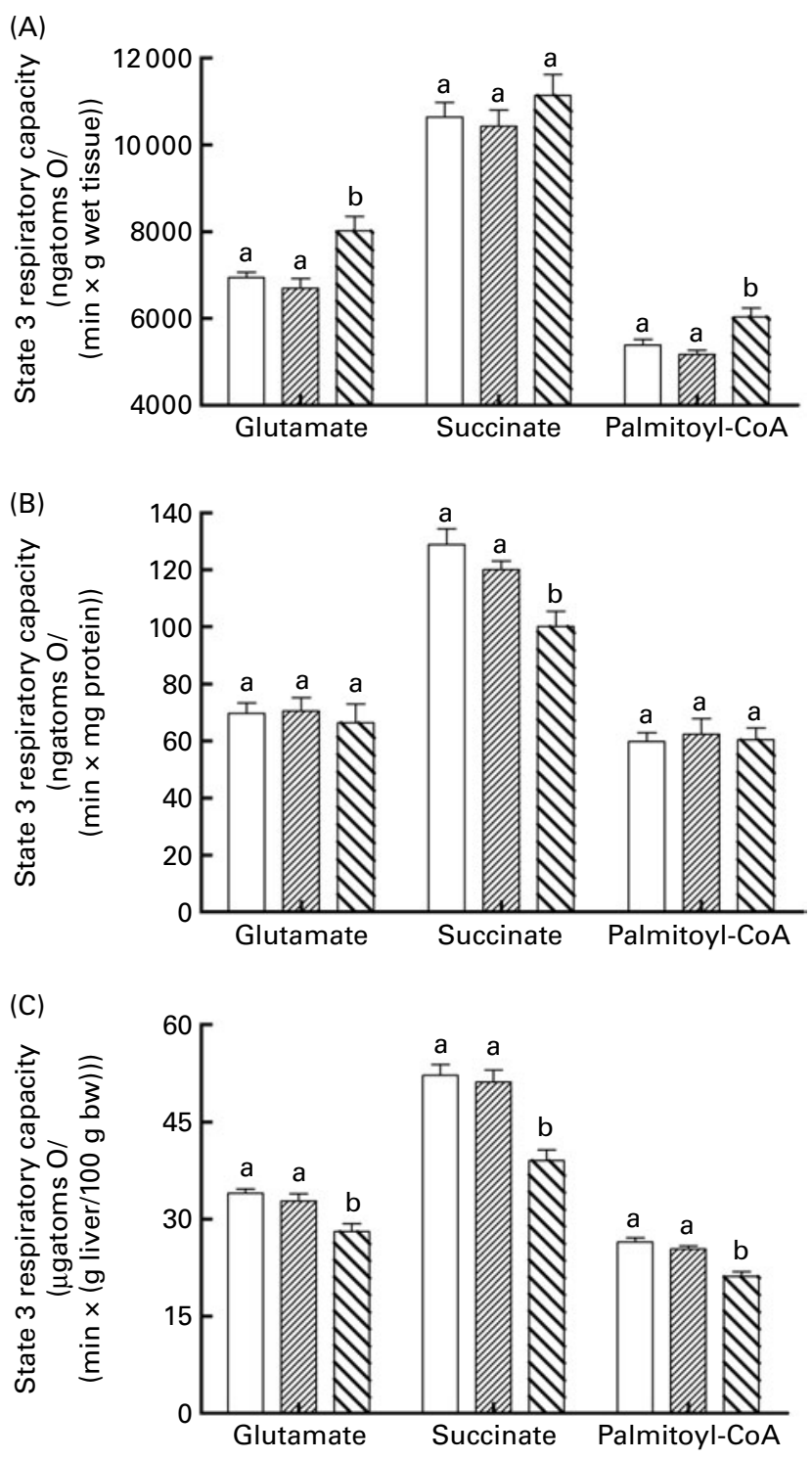

Fig. 2. State 3 respiratory capacities in (A) liver homogenates, $(B)$ isolated mitochondria and (C) per $\mathrm{g}$ liver per $100 \mathrm{~g}$ body weight, with NAD, FAD and lipid substrate in food-restricted and control rats. Values are means, with their standard errors represented by vertical bars of six rats per group. ${ }^{a, b}$ Mean values with unlike letters were significantly different $(P<0.05$; oneway ANOVA followed by Tukey's post-test). WM, weight-matched control rats (); AM, age-matched control rats (四); FR, food-restricted rats $(\mathbf{\mathbb { S }})$.

compared to controls, whereas no variation due to food restriction was found in aconitase activity. The lower oxidative damage in liver mitochondria from FR rats was confirmed by the measurements of lipid peroxidation, showing a significant decrease $(P<0.05)$ in $\mathrm{FR}$ rats compared to controls $(\mathrm{WM}=1.49(\operatorname{sem} 0.06), \mathrm{AM}=1.40($ SEM 0.01$)$ and $\mathrm{FR}=1.16$ (SEM 0.01) nmol of thiobarbituric acid-reactive substances per mg protein).

\section{Discussion}

In the present study, we show that short-term energy restriction in young rats not only decreases energy expenditure but also profoundly affects mitochondrial compartment, both in liver and skeletal muscle. To avoid difficulties in comparing energy expenditure values in rats with different body weight and composition ${ }^{(29,30)}$, the age of the rats ( 7 weeks), as well as the duration (2 weeks) and the degree (50\%) of energy restriction were chosen to significantly decrease lipid stores, while body weight and body proteins remained constant. This allowed us to compare FR rats to WM controls with the same body weight and the same amount of lean, metabolically active, tissue. Under these conditions, therefore, the decreased RMR found in FR rats compared to WM controls reflects adaptive modifications of whole-body energy efficiency. In addition, the decreased $24 \mathrm{~h}$ energy expenditure found in FR rats reflects changes in organ metabolism, as no change in physical activity was induced by food restriction.
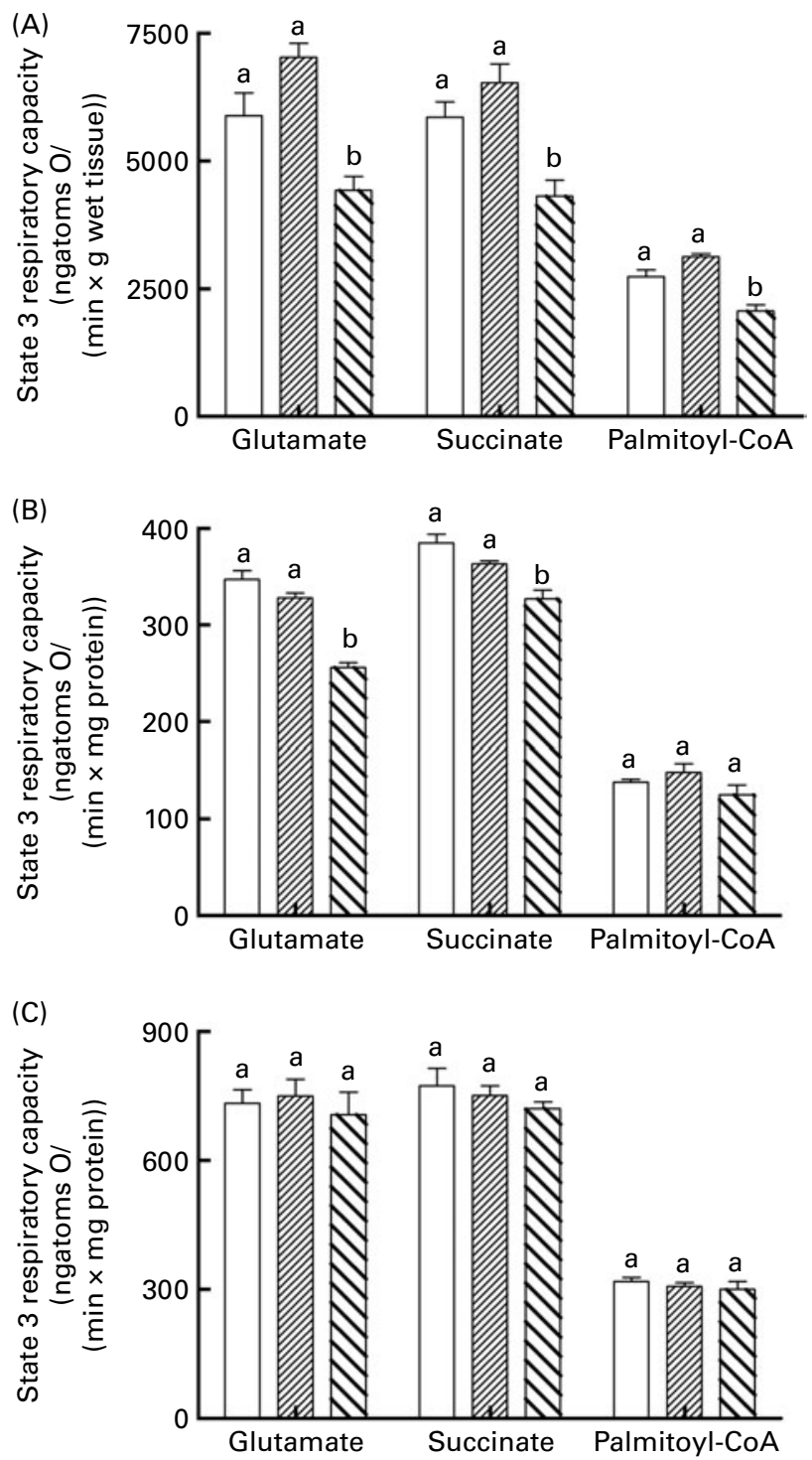

Fig. 3. State 3 respiratory capacities in (A) skeletal muscle homogenates and (B) isolated sub-sarcolemmal and (C) inter-myofibrillar mitochondria, with NAD, FAD and lipid substrate in food-restricted and control rats. Values are means, with their standard errors represented by vertical bars of six rats per group. ${ }^{\mathrm{a}, \mathrm{b}}$ Mean values with unlike letters were significantly different $(P<0.05$; one-way ANOVA followed by Tukey's post-test). WM, weight-matched control rats $(\square)$; AM, age-matched control rats $(\mathbb{0})$; FR, food-restricted rats $(\mathbf{\mathbb { N }})$. 
Table 2. Thermodynamic coupling $(q)$ and optimal thermodynamic efficiency $(\eta)$ of oxidative phosphorylation in hepatic and skeletal muscle mitochondria from food restricted (FR) and control rats

(Mean values with their standard errors of six rats per group)

\begin{tabular}{|c|c|c|c|c|c|c|}
\hline & \multicolumn{2}{|c|}{ WM } & \multicolumn{2}{|c|}{ AM } & \multicolumn{2}{|c|}{ FR } \\
\hline & Mean & SEM & Mean & SEM & Mean & SEM \\
\hline \multicolumn{7}{|l|}{ Liver mitochondria } \\
\hline \multicolumn{7}{|c|}{ Oxygen consumption (ngatoms $\mathrm{O} /(\mathrm{min} \times \mathrm{mg}$ protein)) } \\
\hline + olygomicin & $16 \cdot 5^{\mathrm{a}}$ & 0.5 & $15 \cdot 8^{\mathrm{a}}$ & 0.6 & $19 \cdot 3^{\mathrm{b}}$ & $1 \cdot 0$ \\
\hline + olygomicin + palmitate & $37.4^{\mathrm{a}}$ & $2 \cdot 4$ & $35 \cdot 4^{\mathrm{a}}$ & 1.8 & $29.9^{\mathrm{b}}$ & 0.5 \\
\hline+ FCCP & $148^{\mathrm{a}}$ & 6 & $153^{\mathrm{a}}$ & 6 & $123^{\mathrm{b}}$ & 6 \\
\hline + FCCP + palmitate & $150^{\mathrm{a}}$ & 4 & $151^{\mathrm{a}}$ & 5 & $122^{\mathrm{b}}$ & 5 \\
\hline$q$ & $0.941^{a}$ & 0.004 & $0.946^{a}$ & 0.005 & $0.920^{b}$ & 0.007 \\
\hline$\eta$ & $0.499^{a}$ & 0.009 & $0.517^{\mathrm{a}}$ & 0.016 & $0.439^{b}$ & 0.017 \\
\hline$q_{\text {palmitate }}$ & $0.865^{\mathrm{a}}$ & 0.014 & $0.873^{\mathrm{a}}$ & 0.012 & $0.860^{\mathrm{a}}$ & 0.018 \\
\hline$\eta_{\text {palmitate }}$ & $0.332^{\mathrm{a}}$ & 0.022 & $0.341^{\mathrm{a}}$ & 0.017 & $0.335^{\mathrm{a}}$ & 0.026 \\
\hline \multicolumn{7}{|l|}{ Skeletal muscle SS mitochondria } \\
\hline \multicolumn{7}{|c|}{ Oxygen consumption (ngatoms $\mathrm{O} /(\mathrm{min} \times \mathrm{mg}$ protein)) } \\
\hline + oligomycin & $61.9^{a}$ & 2.9 & $62 \cdot 6^{\mathrm{a}}$ & 4.6 & $56 \cdot 8^{a}$ & $4 \cdot 6$ \\
\hline + oligomycin + palmitate & $68 \cdot 1^{\mathrm{a}}$ & $3 \cdot 2$ & $65 \cdot 4^{\mathrm{a}}$ & $4 \cdot 1$ & $65 \cdot 8^{\mathrm{a}}$ & $6 \cdot 1$ \\
\hline$+\mathrm{FCCP}$ & $385^{\mathrm{a}}$ & 8 & $384^{\mathrm{a}}$ & 10 & $309^{b}$ & 15 \\
\hline + FCCP + palmitate & $389^{a}$ & 7 & $386^{\mathrm{a}}$ & 8 & $312^{\mathrm{b}}$ & 10 \\
\hline$q$ & $0.919^{a}$ & 0.002 & $0.915^{a}$ & 0.002 & $0.907^{b}$ & 0.002 \\
\hline$\eta$ & $0.432^{\mathrm{a}}$ & 0.002 & $0.425^{\mathrm{a}}$ & 0.002 & $0.407^{\mathrm{b}}$ & 0.002 \\
\hline$q_{\text {palmitate }}$ & $0.907^{a}$ & 0.002 & $0.909^{a}$ & 0.002 & $0.875^{\mathrm{b}}$ & 0.002 \\
\hline$\eta_{\text {palmitate }}$ & $0.407^{a}$ & 0.002 & $0.412^{\mathrm{a}}$ & 0.002 & $0.348^{\mathrm{b}}$ & 0.002 \\
\hline \multicolumn{7}{|l|}{ Skeletal muscle IMF mitochondria } \\
\hline \multicolumn{7}{|c|}{ Oxygen consumption (ngatoms $\mathrm{O} /(\min \times \mathrm{mg}$ protein)) } \\
\hline + oligomycin & $124^{\mathrm{a}}$ & 5 & $119^{\mathrm{a}}$ & 1 & $116^{\mathrm{a}}$ & 3 \\
\hline + oligomycin + palmitate & $158^{\mathrm{a}}$ & 8 & $139^{\mathrm{a}}$ & 7 & $145^{\mathrm{a}}$ & 7 \\
\hline+ FCCP & $772^{\mathrm{a}}$ & 21 & $757^{\mathrm{a}}$ & 15 & $802^{\mathrm{a}}$ & 32 \\
\hline + FCCP + palmitate & $780^{\mathrm{a}}$ & 19 & $760^{\mathrm{a}}$ & 12 & $740^{\mathrm{a}}$ & 23 \\
\hline$q$ & $0.915^{\mathrm{a}}$ & 0.002 & $0.913^{a}$ & 0.002 & $0.914^{a}$ & 0.002 \\
\hline$\eta$ & $0.425^{\mathrm{a}}$ & 0.002 & $0.420^{\mathrm{a}}$ & 0.002 & $0.423^{\mathrm{a}}$ & 0.002 \\
\hline$q_{\text {palmitate }}$ & $0.892^{a}$ & 0.002 & $0.899^{a}$ & 0.002 & $0.897^{a}$ & 0.002 \\
\hline$\eta_{\text {palmitate }}$ & $0.377^{a}$ & 0.005 & $0.379^{a}$ & 0.005 & $0.395^{a}$ & 0.005 \\
\hline
\end{tabular}

WM, weight-matched control rats; AM, age-matched control rats; SS, sub-sarcolemmal; IMF, intermyofibrillar.

${ }^{\mathrm{a}, \mathrm{b}}$ Mean values within a row with unlike superscript letters were significantly different $(P<0.05$; one-way ANOVA followed by Tukey's post-test).

Table 3. Cytochrome $c$ content and cytochrome oxidase activity in homogenates and isolated mitochondria from liver and skeletal muscle in food restricted (FR) and control rats

(Mean values with their standard errors of six rats per group)

\begin{tabular}{|c|c|c|c|c|c|c|}
\hline & \multicolumn{2}{|c|}{ WM } & \multicolumn{2}{|c|}{ AM } & \multicolumn{2}{|c|}{ FR } \\
\hline & Mean & SEM & Mean & SEM & Mean & SEM \\
\hline \multicolumn{7}{|l|}{ Liver } \\
\hline Homogenate cytochrome $c$ content (cytochrome $c$ :actin ratio) & $1 \cdot 28^{\mathrm{a}}$ & 0.08 & 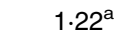 & $0 \cdot 10$ & $1.64^{\mathrm{b}}$ & 0.10 \\
\hline Homogenate COX activity ( $\mu$ gatoms $\mathrm{O} /(\min \times \mathrm{g}$ tissue $))$ & $66^{\mathrm{a}}$ & 5 & $61^{\mathrm{a}}$ & 2 & $77^{\mathrm{b}}$ & 4 \\
\hline Mitochondrial COX activity $(\mu$ gatoms $\mathrm{O} /(\min \times \mathrm{g}$ tissue $))$ & $10 \cdot 7^{\mathrm{a}}$ & 0.9 & $9 \cdot 8^{\mathrm{a}}$ & 0.8 & $12 \cdot 1^{\mathrm{b}}$ & $1 \cdot 1$ \\
\hline Mitochondrial COX activity (ngatoms $\mathrm{O} /(\mathrm{min} \times \mathrm{mg}$ protein)) & $1008^{\mathrm{a}}$ & 74 & $890^{\mathrm{a}}$ & 72 & $979^{\mathrm{a}}$ & 45 \\
\hline Mitochondrial protein yield (mg/g tissue) & $10 \cdot 6^{\mathrm{a}}$ & 1.0 & $11 \cdot 0^{\mathrm{a}}$ & 0.9 & $12 \cdot 3^{\mathrm{b}}$ & 0.9 \\
\hline COX recovery (\%) & $16^{\mathrm{a}}$ & 1 & $16^{\mathrm{a}}$ & 1 & $16^{\mathrm{a}}$ & 1 \\
\hline \multicolumn{7}{|l|}{ Skeletal muscle } \\
\hline Homogenate cytochrome $c$ content (cytochrome $c$ :actin ratio) & $1 \cdot 17^{\mathrm{a}}$ & 0.08 & $1 \cdot 19^{\mathrm{a}}$ & 0.08 & $0.90^{\mathrm{b}}$ & 0.05 \\
\hline Homogenate COX activity ( $\mu$ gatoms $\mathrm{O} /(\min \times \mathrm{g}$ tissue $))$ & $35 \cdot 2^{\mathrm{a}}$ & $2 \cdot 0$ & $37 \cdot 9^{\mathrm{a}}$ & $2 \cdot 0$ & $24 \cdot 5^{b}$ & $2 \cdot 0$ \\
\hline IMF Mitochondrial COX activity (ngatoms $\mathrm{O} /(\min \times \mathrm{g}$ tissue)) & $7944^{\mathrm{a}}$ & 352 & $7608^{a}$ & 289 & $5040^{\mathrm{b}}$ & 270 \\
\hline IMF Mitochondrial COX activity (ngatoms $\mathrm{O} /(\min \times \mathrm{mg}$ protein) $)$ & $3783^{\mathrm{a}}$ & 250 & $3458^{\mathrm{a}}$ & 210 & $3600^{\mathrm{a}}$ & 222 \\
\hline IMF Mitochondrial protein yield (mg/g tissue) & $2 \cdot 1^{\mathrm{a}}$ & $0 \cdot 1$ & $2 \cdot 2^{\mathrm{a}}$ & $0 \cdot 1$ & $1.4^{\mathrm{b}}$ & 0.1 \\
\hline IMF COX recovery $(\%)$ & $22 \cdot 6^{a}$ & $1 \cdot 2$ & $20 \cdot 1^{a}$ & $1 \cdot 2$ & $20 \cdot 1^{\mathrm{a}}$ & $1 \cdot 0$ \\
\hline SS Mitochondrial COX activity (ngatoms $\mathrm{O} /(\min \times \mathrm{g}$ tissue $)$ ) & $2144^{\mathrm{a}}$ & 133 & $2511^{\mathrm{a}}$ & 150 & $1234^{\mathrm{b}}$ & 55 \\
\hline SS Mitochondrial COX activity (ngatoms $\mathrm{O} /(\mathrm{min} \times \mathrm{mg}$ protein)) & $4244^{\mathrm{a}}$ & 201 & $4565^{\mathrm{a}}$ & 188 & $3982^{\mathrm{a}}$ & 125 \\
\hline SS Mitochondrial protein yield (mg/g tissue) & $0.51^{a}$ & 0.02 & $0.55^{\mathrm{a}}$ & 0.03 & $0.31^{b}$ & 0.02 \\
\hline SS COX recovery $(\%)$ & $6 \cdot 1^{\mathrm{a}}$ & 0.3 & $6 \cdot 6^{\mathrm{a}}$ & 0.3 & $5 \cdot 1^{\mathrm{a}}$ & 0.3 \\
\hline
\end{tabular}

WM, weight-matched control rats; AM, age-matched control rats; COX, cytochrome oxidase; IMF, intermyofibrillar; SS, sub-sarcolemmal.

${ }^{a, b}$ Mean values within a row with unlike superscript letters were significantly different $(P<0 \cdot 05$; one-way ANOVA followed by Tukey's post-test). 

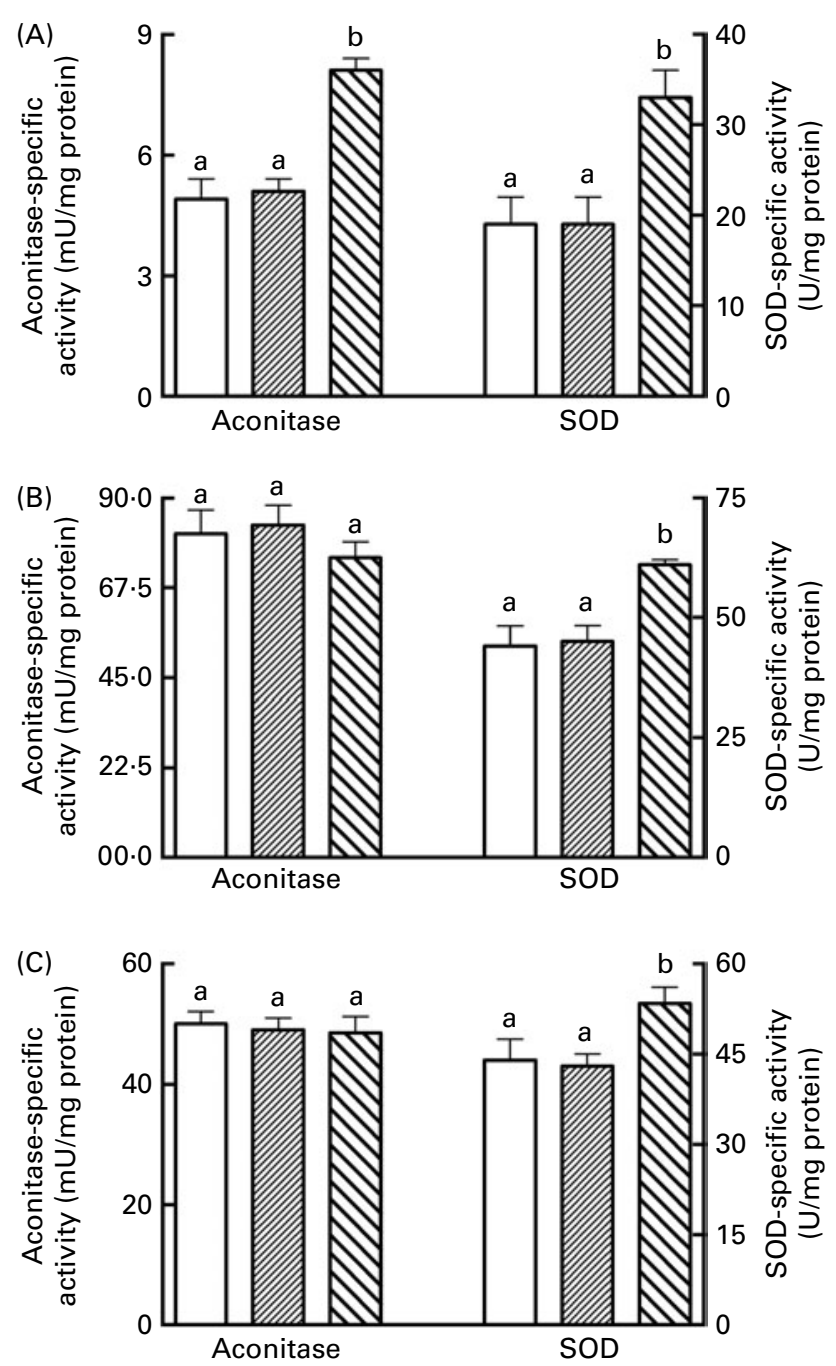

Fig. 4. Aconitase- and superoxide dismutase (SOD)-specific activity in (A) liver and (B) sub-sarcolemmal and (C) inter-myofibrillar skeletal muscle mitochondria in food-restricted and control rats. Values are means, with their standard errors represented by vertical bars of six rats per group. ${ }^{\mathrm{a}, \mathrm{b}}$ Mean values with unlike letters were significantly different $(P<0.05$; one-way ANOVA followed by Tukey's post-test). WM, weight-matched control rats ( $\square$ ); AM, age-matched control rats (司); FR, food-restricted rats $(\mathbb{\mathbf { Q }})$.

Determination of $24 \mathrm{~h} \mathrm{VO}_{2}$ and $\mathrm{VCO}_{2}$ also allows calculation of RQ and non-protein RQ, parameters linked to mixture of oxidised fuels in the cells. The results found here are suggestive of a change in the use of fuels from glucose to fat in FR rats, independently of the light-dark phase. In addition, the value of non-protein RQ $>1$ found in controls indicates the use of carbohydrate not only for oxidation purposes but also for net 'de novo' lipogenesis ${ }^{(31)}$, while non-protein RQ was found to be 0.91 (SEM 0.04) in FR rats, indicating that energy for maintenance was derived from glucose (70.7\%) and fat (29.3\%), as calculated from Elia \& Livesey ${ }^{(31)}$ and that net 'de novo' lipogenesis was abolished, in agreement with previous findings showing down-regulation of hepatic lipogenic gene expression after 1 week of energy restriction ${ }^{(32)}$.

Another consequence of energy restriction is the significant decrease in body fat, skeletal muscle lipid mass and plasma
NEFA, which could play a role in the beneficial effect of energy restriction, as higher body fat and plasma NEFA levels have been directly implicated in metabolic diseases such as insulin resistance and diabetes ${ }^{(33,34)}$. Lower plasma NEFA at the end of energy restriction could be partly due to the fact that FR rats oxidise more fatty acids than controls, as indicated by lower RQ values both in light and dark phase.

As for the liver, the present results indicate a significant decrease in isolated mitochondria oxidative capacity with FAD-linked but not NAD-linked and lipid substrate. A significant decrease was also found in maximal uncoupled respiration, thus allowing us to exclude the system of ATP synthesis, such as ATP synthetase and ATP/ADP carrier, and suggesting an impairment at the level of the respiratory chain from complex II onwards, as also found by others after $3 \mathrm{~d}$ of food restriction ${ }^{(35)}$. On the other hand, a significant increase in mitochondrial protein mass was found in FR rats, in agreement with other studies after long-term energy restriction in young or old rats ${ }^{(36,37)}$, so that when respiratory capacity was assessed in homogenates, to take into account mass and activity of mitochondria, significantly higher respiratory capacity was found with NAD-linked and lipid, but not with FAD-linked, substrates. An increase in mitochondrial mass could be useful to allow the metabolic shift from carbohydrate to fat, which occurs in FR rats, as mitochondrial biogenesis is induced when fatty acids are the main energy fuels ${ }^{(38)}$. In addition, higher mitochondrial mass could supply ATP needed to support increased gluconeogenesis found in FR rats ${ }^{(39)}$. Accordingly, we have found no variation in hepatic glycogen content, although carbohydrate intake in FR rats was $50 \%$ lower than in control rats.

When hepatic respiratory capacities were referred to $100 \mathrm{~g}$ body weight, significantly lower values were obtained in FR rats compared to controls. In fact, we found a significant decrease in liver weight, both as absolute values and as percent of body weight in FR rats compared to controls, indicating that food restriction selectively reduces liver weight. As the liver plays a primary metabolic role, the aforementioned decline in tissue mass could have a deep impact on whole-body energy homostasis.

Another important factor that influences mitochondrial ATP production is the degree of coupling of oxidative phosphorylation, which was found unchanged in liver from FR rats, when measurements were done in the presence of physiological amounts of fatty acid palmitate, to take into account uncoupling effect of fatty acids. This result is in agreement with Ramsey et $a l .{ }^{(40)}$, who found no variation in hepatic proton leak, a major contributor to mitochondrial degree of coupling, after 1 and 6 months of energy restriction. However, as the degree of coupling also depends on cellular fatty acid concentrations, which are likely to be dictated by the corresponding concentrations in the plasma ${ }^{(41)}$, the finding of lower NEFA plasma levels in FR rats suggests that the degree of coupling is higher in FR rats. A higher mitochondrial coupling would cause less substrate to be oxidised in order to meet cellular energy demand, and therefore energy saving.

The mitochondria are also the main source of ROS, hence being highly exposed to oxidative stress ${ }^{(10)}$. However, hepatic 
mitochondria from FR rats showed a lower degree of oxidative stress, probably because of the increased antioxidant SOD activity, which could be one of the mechanisms at the basis of the beneficial effect of energy restriction on oxidative stress $^{(4)}$, at least in the liver.

The present study also indicated that, in skeletal muscle, IMF and SS mitochondrial protein mass per $g$ of tissue was significantly reduced in FR rats, compared to controls. In line with this result, significantly lower state 3 respiratory capacity was found in skeletal muscle homogenate. The decreased skeletal muscle mitochondrial protein mass could be due to decreased plasma NEFA levels found in FR rats, as it has been shown that plasma NEFA concentrations affect skeletal muscle mitochondrial biogenesis, probably through $\operatorname{PPAR} \delta^{(42)}$. In addition, SS mitochondria displayed lower uncoupled respiration and state 3 respiratory capacity with succinate or glutamate as substrate, indicating an impairment of the respiratory chain and not of the system involved in ATP synthesis. IMF mitochondria isolated from FR rats also exhibited a significantly higher degree of coupling when measurements were done in the presence of physiological amounts of fatty acid palmitate, which is in agreement with Bevilacqua et al. ${ }^{(43)}$, who found a decrease in skeletal muscle proton leak after short- and long-term energy restriction. Therefore, it can be hypothesised that skeletal muscle cells in FR rats consume less oxygen, because of their lower mitochondrial content, and obtain ATP from energy substrates at higher efficiency, because of the higher $q$. The combination of these two adaptive modifications would contribute to energy sparing elicited by food restriction, as skeletal muscle is a significant contributor to whole-body energy utilisation $^{(11)}$. In fact, higher IMF mitochondrial efficiency implies that less amount of fuels are burned to obtain the same amount of ATP needed to support unchanged physical activity of FR rats. A surprising result was the decreased degree of coupling found in SS mitochondria from FR rats compared to controls. This finding can be explained taking into account that SS mitochondria also display lower rates of state 3 respiration, so that, because of the reduced activity of respiratory chain, they operate in a condition in which proton leak contribution to total respiration is higher than in control rats and hence degree of coupling is lower ${ }^{(44)}$. However, the decreased degree of coupling in SS mitochondria from FR rats could be balanced by the lower plasma NEFA levels.

Several works have studied the effect of energy restriction on skeletal muscle mitochondrial bioenergetics, and the results are somewhat conflicting, because of the great variability in the age of the rats, the duration of the food-restriction period and the dynamics of weight changes ${ }^{(32,45-48)}$. Barazzoni et al. ${ }^{(32)}$ found increased skeletal muscle mitochondrial oxidative capacity in response to energy restriction. However, this result has been obtained after 3 weeks of $26 \%$ food restriction and using rats older than those in the present study. On the other hand, younger (about 8 weeks) rats FR for 2 weeks exhibited a significant decrease in oxidative capacity of mixed muscle ${ }^{(45)}$, although this effect was not evident after a longer restriction period $^{(48)}$.
An important physiological implication of increased degree of coupling in IMF mitochondria from FR rats could be an increased ROS production, especially at rest, when skeletal muscle energy turnover is low and mitochondrial respiration operates near state 4 conditions ${ }^{(49)}$. However, the potential harmful consequence of increasing mitochondrial coupling is counteracted by increasing cellular antioxidant defences. The present result is in agreement with previous ones showing no effect of short-term energy restriction on skeletal muscle mitochondrial $\mathrm{H}_{2} \mathrm{O}_{2}$ production ${ }^{(47,50)}$, but do not agree with those reported by Bevilacqua et al. ${ }^{(43)}$, who found lower $\mathrm{H}_{2} \mathrm{O}_{2}$ production in isolated skeletal muscle mitochondria after 2 weeks of $60 \%$ energy restriction. However, measurement of the present study reflects 'in vivo' oxidative damage due to ROS production, whereas $\mathrm{H}_{2} \mathrm{O}_{2}$ measurements were indicative of 'in vitro' maximal capacity for mitochondrial ROS production.

In conclusion, from the present results, it appears that in liver and skeletal muscle, which account for 20 and $30 \%$, respectively, of whole-body resting energy expenditure in the rat ${ }^{(11)}$, mitochondrial compartment is differently affected by energy restriction. In fact, in liver, mitochondrial mass increases in response to energy restriction, while the metabolically active tissue mass is reduced. On the other hand, skeletal muscle mitochondrial activity (SS) and mass (SS and IMF) decrease, while IMF mitochondrial efficiency rises, thus contributing to energy sparing in this tissue.

\section{Acknowledgements}

The present study was supported by a grant from the University 'Federico II' of Naples and by grant no. 31-130481 from the Swiss National Science Foundation. The authors thank Emilia De Santis for skilful management of the animal house. S. I. designed and supervised the study. S. I., G. L. and A. G. D. obtained funding and provided administrative, technical and material support. R. C., F. B., I. F. and P. C. performed the animal experiments. R. C. and S. I. contributed to the analysis of data and interpretation of the results. R. C., S. I. and G. L. wrote the draft of the manuscript, and all the authors critically reviewed the manuscript. The authors declare no conflicts of interest.

\section{References}

1. Ramsey JJ, Harper ME \& Weindruch R (2000) Restriction of energy intake, energy expenditure and aging. Free Radic Biol Med 29, 946-968.

2. Barzilai N \& Gabriely I (2001) The role of fat depletion in the biological benefits of caloric restriction. I Nutr $\mathbf{1 3 1}$ 903S-906S.

3. Anderson RM \& Weindruch R (2010) Metabolic reprogramming, caloric restriction and aging. Trends Endocrinol Metab 21, 134-141.

4. Gredilla R \& Barja G (2005) The role of oxidative stress in relation to caloric restriction and longevity. Endocrinology 146, 3713-3717.

5. Lanza IR \& Nair KS (2010) Mitochondrial function as a determinant of life span. Pflug Arch 459, 277-289. 
6. Harper ME, Green K \& Brand MD (2008) The efficiency of cellular energy transduction and its implications for obesity. Ann Rev Nutr 28, 13-33.

7. Stucki JW (1980) The optimal efficiency and the economic degrees of coupling of oxidative phosphorylation. Eur J Biochem 109, 269-283.

8. Skulachev VP (1991) Fatty acid circuit as a physiological mechanism of uncoupling of oxidative phosphorylation. FEBS Lett 294, 158-162.

9. Jezek P, Engstova H, Zackova M, et al. (1998) Fatty acid cycling mechanism and mitochondrial uncoupling proteins. Biochim Biophys Acta 1365, 319-327.

10. Brand MD (2010) The sites and topology of mitochondrial superoxide production. Exp Gerontol 45, 466-472.

11. Rolfe DFS \& Brown GC (1997) Cellular energy utilization and molecular origin of standard metabolic rate in mammals. Physiol Rev 77, 731-758.

12. Cogswell AM, Stevens RJ \& Hood DA (1993) Properties of skeletal muscle mitochondria isolated from subsarcolemmal and intermyofibrillar regions. Am J Physiol 264, C383-C389.

13. Mollica MP, Lionetti L, Crescenzo R, et al. (2006) Heterogeneous bioenergetic behaviour of subsarcolemmal and intermyofibrillar mitochondria in fed and fasted rats. Cell Mol Life Sci 63, 358-366.

14. Crescenzo R, Lionetti L, Mollica MP, et al. (2006) Altered skeletal muscle subsarcolemmal mitochondrial compartment during catch-up fat after caloric restriction. Diabetes $\mathbf{5 5}$, 2286-2293.

15. Lionetti L, Mollica MP, Crescenzo R, et al. (2007) Skeletal muscle subsarcolemmal mitochondrial dysfunction in high-fat fed rats exhibiting impaired glucose homeostasis. Int J Obes 31, 1596-1604.

16. Iossa S, Lionetti L, Mollica MP, et al. (1999) Energy intake and utilization vary during development in rats. $J$ Nutr $\mathbf{1 2 9}$, 1593-1596.

17. Folch J, Lees M \& Sloane Stanley GH (1957) A simple method for the isolation and purification of total lipides from animal tissues. J Biol Chem 226, 497-510.

18. Dulloo AG \& Girardier L (1992) Influence of dietary composition on energy expenditure during recovery of body weight in the rat: implications for catch-up growth and obesity relapse. Metabolism 41, 1336-1342.

19. Armsby HP (1917) The Nutrition of Farm Animals. New York, NY: The Macmillan Company.

20. Roehrig KL \& Allred JB (1974) Direct enzymatic procedure for the determination of liver glycogen. Anal Biochem 58, 414-421.

21. Iossa S, Lionetti L, Mollica MP, et al. (2003) Effect of high-fat feeding on metabolic efficiency and mitochondrial oxidative capacity in adult rats. Br J Nutr 90, 953-960.

22. Iossa S, Mollica MP, Lionetti L, et al. (2002) Skeletal muscle oxidative capacity in rats fed high-fat diet. Int $\mathrm{J}$ Obes $\mathbf{2 6}$, 65-72.

23. Cairns CB, Walther J, Harken AH, et al. (1998) Mitochondrial oxidative phosphorylation efficiencies reflect physiological organ roles. Am J Physiol 274, R1376-R1383.

24. Barré H, Bailly L \& Rouanet JL (1987) Increased oxidative capacity in skeletal muscle from cold-acclimated ducklings: a comparison with rats. Comp Biochem Physiol 88B, 5119-5522.

25. Crescenzo R, Bianco F, Falcone I, et al. (2010) Hepatic mitochondrial energetics during catch-up fat after caloric restriction. Metabolism 59, 1221-1230.

26. Gardner PR (2002) Aconitase: sensitive target and measure of superoxide. Meth Enzymol 349, 9-16.
27. Fernandes MAS, Custodio JBA, Santos MS, et al. (2006) Tetrandrine concentrations not affecting oxidative phosphorylation protect rat liver mitochondria from oxidative stress. Mitochondrion 6, 176-185.

28. Flohè L \& Otting F (1984) Superoxide dismutase assay. Meth Enzymol 105, 93-104.

29. Dulloo AG \& Girardier L (1993) 24 hour energy expenditure several months after weight loss in the underfed rat: evidence for a chronic increase in whole-body metabolic efficiency. Int J Obes Relat Metab Disord 17, 115-123.

30. Crescenzo R, Samec S, Antic V, et al. (2003) A role for suppressed thermogenesis favouring catch-up fat in the pathophysiology of catch-up growth. Diabetes 52, 1090-1097.

31. Elia M \& Livesey G (1988) Theory and validity of indirect calorimetry during net lipid synthesis. Am J Clin Nutr $\mathbf{4 7}$, 591-607.

32. Barazzoni R, Zanetti M, Bosutti A, et al. (2005) Moderate caloric restriction, but not physiological hyperleptinemia per se, enhances mitochondrial oxidative capacity in rat liver and skeletal muscle - tissue specific impact on tissue triglyceride content and AKT activation. Endocrinol 146, 2098-2106.

33. Virtue S \& Vidal-Puig A (2010) Adipose tissue expandability, lipotoxicity and the metabolic syndrome - an allostatic perspective. Biochim Biophys Acta 1801, 338-349.

34. Boden G \& Shulman GI (2002) Free fatty acids in obesity and type 2 diabetes: defining their role in the development of insulin resistance and beta-cell dysfunction. Eur J Clin Invest 32, 14-23

35. Dumas JF, Roussel D, Simard G, et al. (2004) Food restriction affects energy metabolism in rat liver mitochondria. Biochim Biophys Acta 1670, 126-131.

36. Lambert AJ, Wang B, Yardley J, et al. (2004) The effect of aging and caloric restriction on mitochondrial protein density and oxygen consumption. Exp Gerontol 39, 289-295.

37. López-Lluch G, Hunt N, Jones B, et al. (2006) Calorie restriction induces mitochondrial biogenesis and bioenergetic efficiency. Proc Natl Acad Sci U S A 103, 1768-1773.

38. Wallace DC, Fan W \& Procaccio V (2010) Mitochondrial energetics and therapeutics. Annu Rev Pathol 5, 297-348.

39. Hagopian K, Ramsey JJ \& Weindruch R (2003) Caloric restriction increases gluconeogenic and transaminase enzyme activities in mouse liver. Exp Gerontol 38, 267-278.

40. Ramsey JJ, Hagopian K, Kenny TM, et al. (2004) Proton leak and hydrogen peroxide production in liver mitochondria from energy-restricted rats. Am J Physiol Endocrinol Metab 286, E31-E40.

41. Soboll S \& Stucki J (1985) Regulation of the degree of coupling of oxidative phosphorylation in intact rat liver. Biochim Biophys Acta 807, 245-254.

42. Garcia-Roves P, Huss JM, Han DH, et al. (2007) Raising plasma fatty acid concentration induces increased biogenesis of mitochondria in skeletal muscle. Proc Natl Acad Sci US A 104, 10709-10713.

43. Bevilacqua L, Ramsey JJ, Hagopian K, et al. (2004) Effects of short- and medium-term calorie restriction on muscle mitochondrial proton leak and reactive oxygen species production. Am J Physiol 286, E852-E861.

44. Hafner RP, Brown GC \& Brand MD (1990) Analysis of the control of respiration rate, proton leak rate and protonmotive force in isolated mitochondria using the "top-down" approach of metabolic control theory. Eur J Biochem 188, 313-319. 
45. You YN, Short KR, Jourdan M, et al. (2009) The effect of high glucocorticoid administration and food restriction on rodent skeletal muscle mitochondrial function and protein metabolism. PLoS One 4, e5283.

46. Sreekumar R, Unnikrishnan J, Fu A, et al. (2002) Effects of caloric restriction on mitochondrial function and gene transcripts in rat muscle. Am J Physiol 283, E38-E43.

47. Drew B, Phaneuf S, Dirks A, et al. (2003) Effect of aging and caloric restriction on mitochondrial energy production in gastrocnemius muscle and heart. Am J Physiol 284, R474-R480.
48. Hancock CR, Han DH, Higashida K, et al. (2011) Does calorie restriction induce mitochondrial biogenesis? A reevaluation. FASEB J 25, 785-791.

49. Korshunov SS, Skulachev VP \& Starkov AA (1997) High protonic potential actuates a mechanism of production of reactive oxygen species in mitochondria. FEBS Lett $\mathbf{4 1 6}$ 15-18.

50. Gredilla R, Phaneuf S, Selman C, et al. (2004) Short-term caloric restriction and sites of oxygen radical generation in kidney and skeletal muscle mitochondria. Ann N Y Acad Sci 1019, 333-342. 